\title{
GENEESKUNDIGE WOORDENLIJST.
}




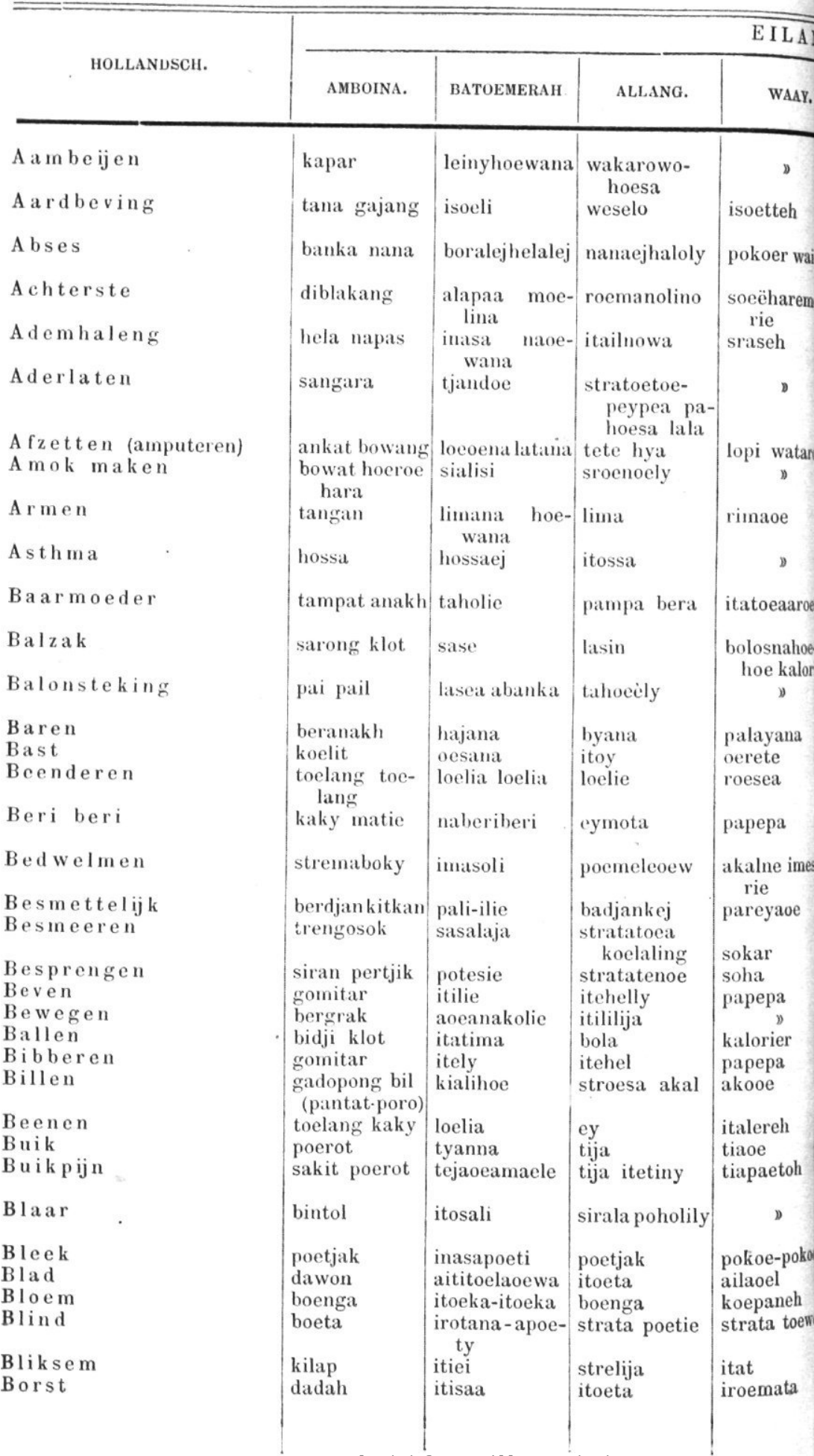




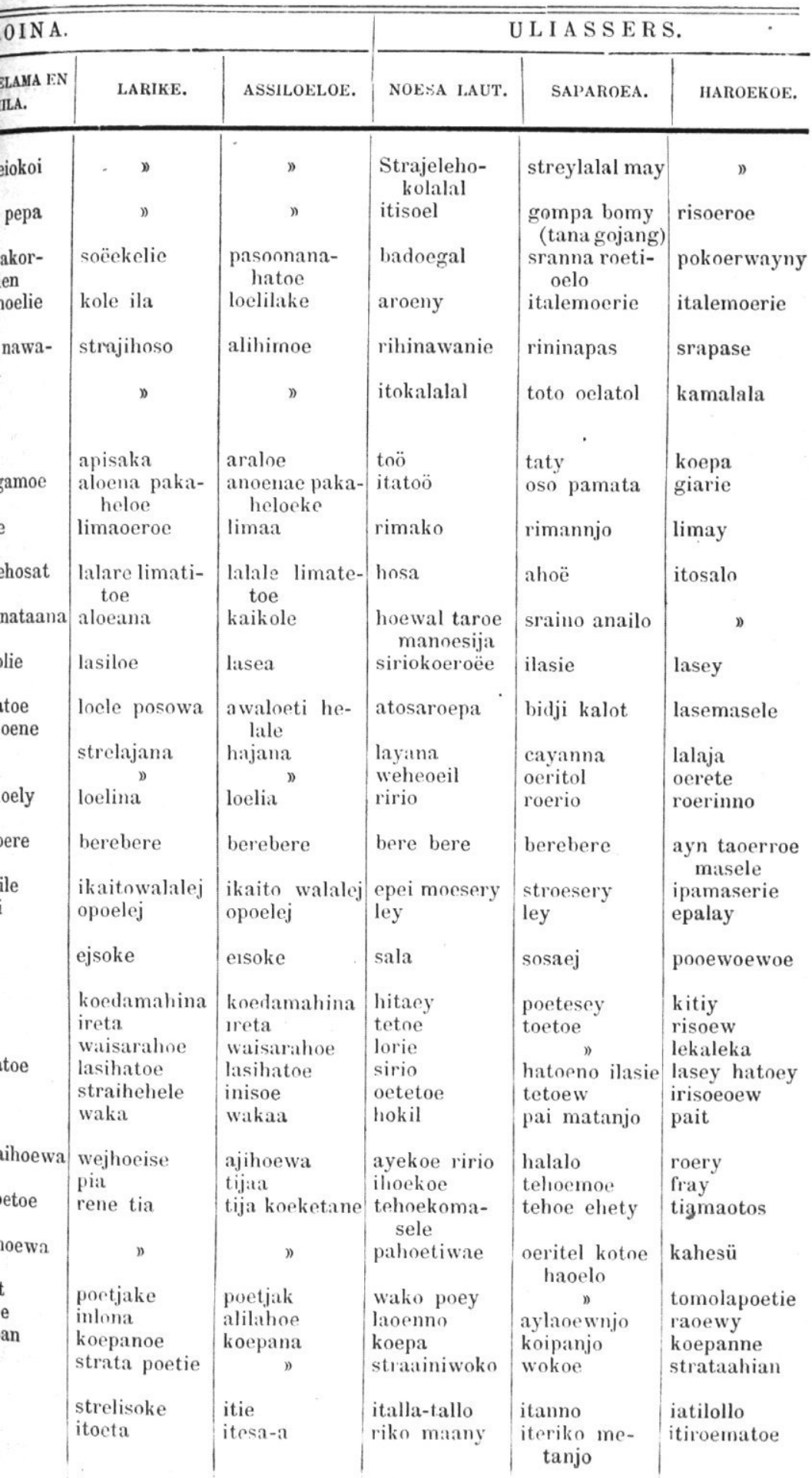




\begin{tabular}{|c|c|c|c|c|}
\hline \multirow{2}{*}{ HOLLANDSCH. } & \multicolumn{2}{|c|}{ B O E ROE } & \multicolumn{2}{|c|}{ N. $0 . \mathrm{K}$ U S T $V$} \\
\hline & KAJELI. & ALEFOEROE. & нотті. & HATO \\
\hline $\begin{array}{l}\text { A ambeijen } \\
\text { A ardbeving }\end{array}$ & $\begin{array}{l}\text { oelama fbare- } \\
\text { toe walama } \\
\text { iso }\end{array}$ & $\begin{array}{l}\text { oelama (bere- } \\
\text { betapapany) }\end{array}$ & $\begin{array}{l}\text { strantoka } \\
\text { issiboia }\end{array}$ & $\begin{array}{l}\text { koppapoe } \\
\text { hiriass? }\end{array}$ \\
\hline Absces & banana & boela & $n$ & \\
\hline Achterste & parine & pohine & $n$ & 》 \\
\hline A demhaling & iresni napas & daesane napas & oehonnawata & lan \\
\hline Aderlaten & singara & $n$ & bofa oeata & issioelat \\
\hline Afzetten (amputeren) & bosoake & bastelake & 》 & 》 \\
\hline Amok maken & stramoke & damamok & larinsa & strengar \\
\hline Armen & limane & vahane & phalanoean & sriman \\
\hline Asthma & palare & gete & boehaboeha & kapalaa \\
\hline Baarmoeder & anajoboy & sranatelenej & soiphata & kalamala \\
\hline Balzak & tobonoesany & kelan mian & lissiero & taoen \\
\hline Bal ontsteking & tabon nepaso & tafoen poso & lissien bossita & lahoewan \\
\hline Baren & sropanay & daana & strahiwa & $\begin{array}{l}\text { roeilahaa- } \\
\text { lakki }\end{array}$ \\
\hline Bast & oesane & kolin & lana & oeloen \\
\hline Beenderen & lolin lolin & rohin rohin & ririen & loenoe \\
\hline Beri beri & bogboga & bogbaga & $\begin{array}{l}\text { ellenpallolas- } \\
\text { sa }\end{array}$ & rizien \\
\hline Bedwelmen & $\begin{array}{l}\text { enhoeana } \\
\text { manseli }\end{array}$ & $\begin{array}{l}\text { daloea nan- } \\
\text { sihej }\end{array}$ & stroesan & stroesinni \\
\hline Besmettelijk & inlay & dadakie & . & ) \\
\hline Besmeeren & sosa & dasosa & jassie & itakky ak \\
\hline Besprengen & ensona & dasoha & phoerratatta & kassipippi \\
\hline Beven & pepene & dagege & djaadjaphane & kalaloeloe \\
\hline Bewegen & gergera & geda geda & korakain & isoen \\
\hline $\begin{array}{l}\text { Ballen } \\
\text { Bibberen } \\
\text { Billen }\end{array}$ & $\begin{array}{l}\text { tabon batone } \\
\text { pepene } \\
\text { parine }\end{array}$ & $\begin{array}{l}\text { tapon koline } \\
\text { dagige } \\
\text { pohine }\end{array}$ & $\begin{array}{l}\text { lissien } \\
\text { djaadjaphane } \\
\text { strane }\end{array}$ & $\begin{array}{l}\text { taoen oes } \\
\text { kalaloe } \\
\text { topoen }\end{array}$ \\
\hline Beenen & bitiej & kadang & ellen & ain \\
\hline Buik & tiane & tian & toean & tian \\
\hline Buikpijn & poro tianbere & tianpy & D & 》 \\
\hline Bla ar & $n$ & $n$ & pona & titi \\
\hline Bleek & $\underset{\text { jak }}{\text { oehan \& poet- }}$ & poepangawa & kassibitti & kapoeti \\
\hline Blad & tetone & oemoene & riti-inta & tottoen \\
\hline Bloem & tatane & daptata & oemina & aoeloin \\
\hline Blind & inkiso & dakiso & boeran & stratae \\
\hline Bliksem & rraite & daiti & pairatte & hihinalan \\
\hline Borst & srowene & srue-oen & lokkon & inoematts \\
\hline
\end{tabular}












SCHE'S VAN DE RESIDENTIE AMBOINA.

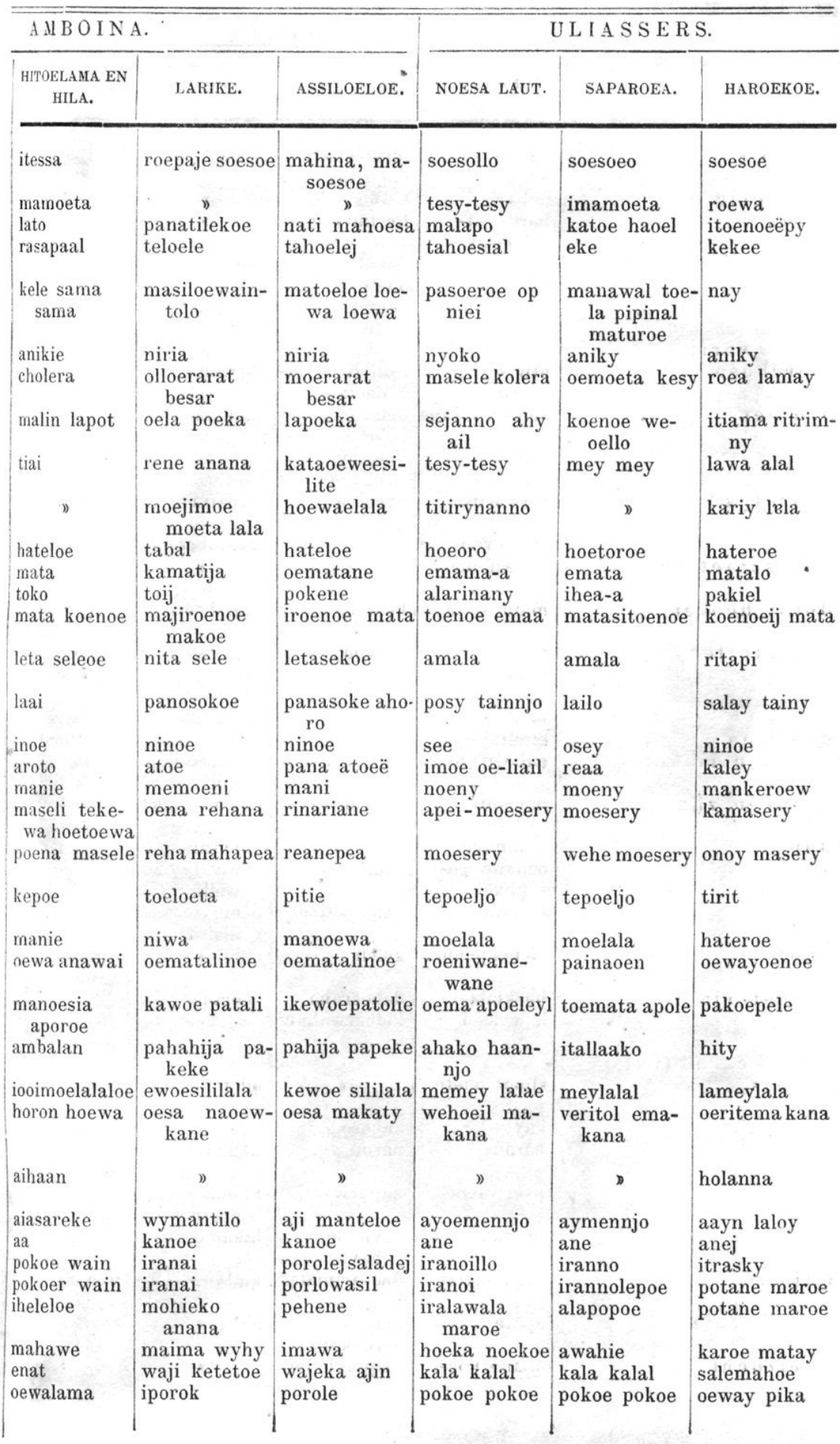




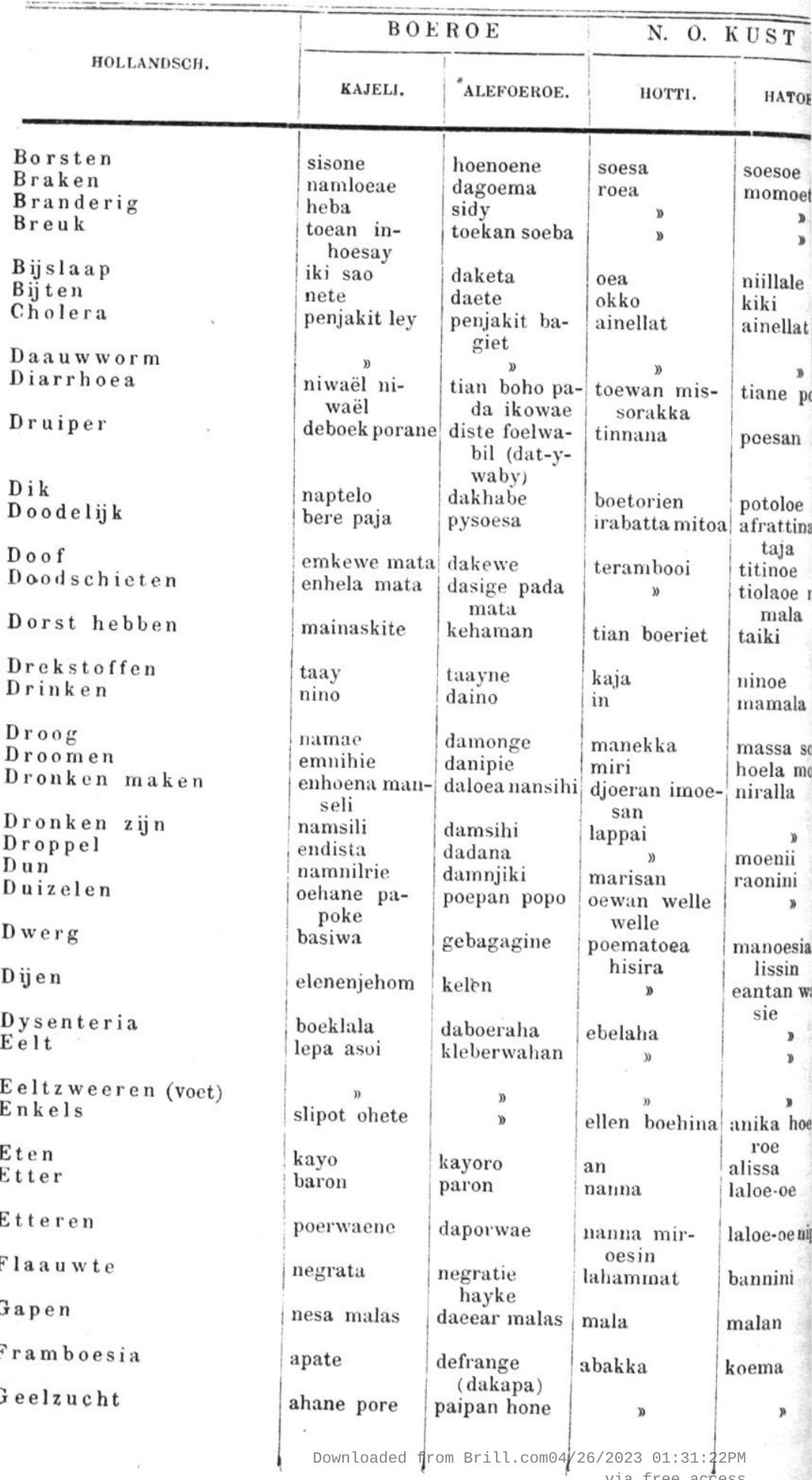




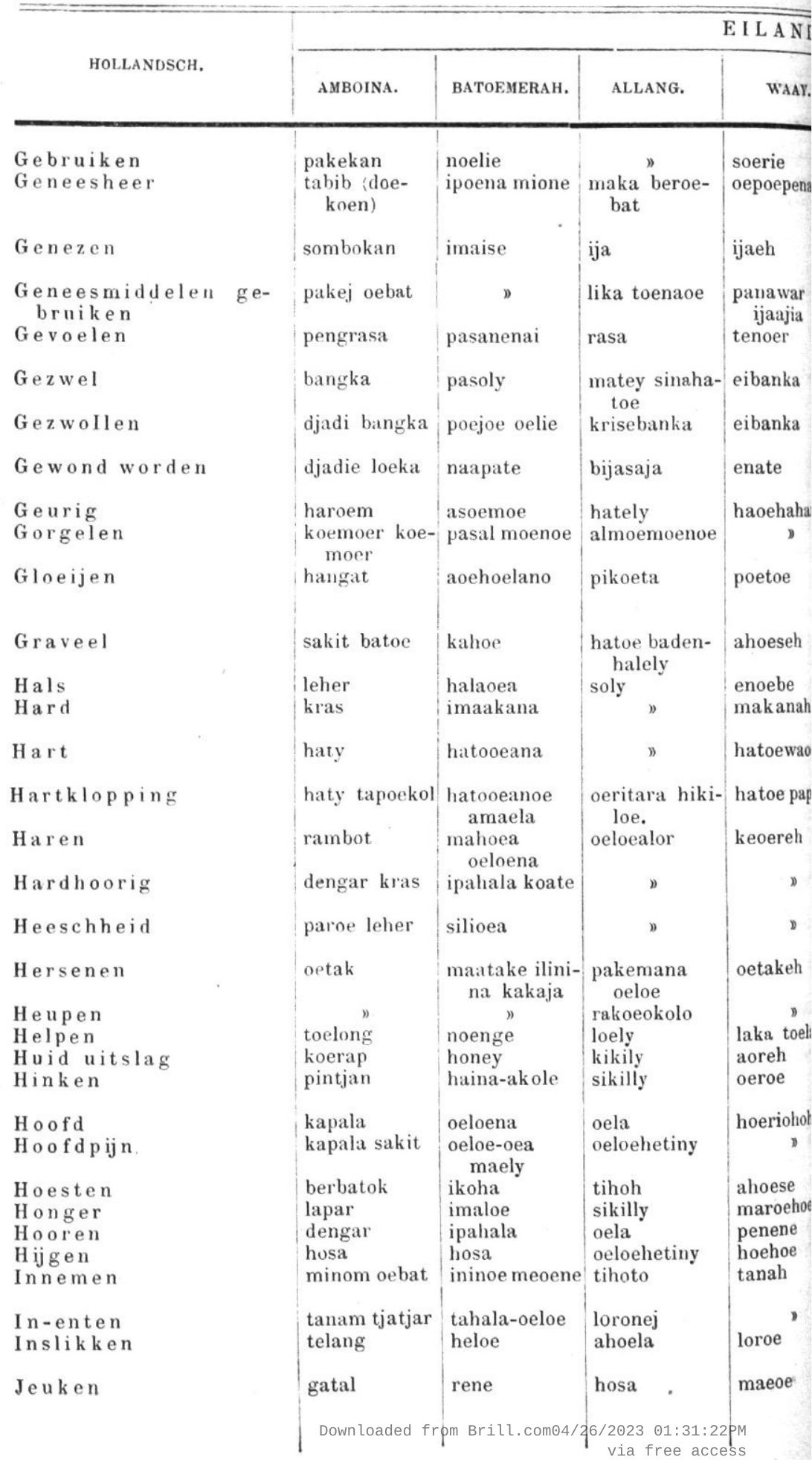




\section{IIBOIN A.}

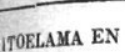

HILA.

oelie

nanoesia

maameoen

joa

enamewoen

asa
oto
oto

oenanak

aoematelle

aramoemoe

angat

hoeseh

noe

rakana

sso

atwaie-

papepa

eoel

ahalateia

D

tak

ssaa

ahale

askadoe

ere

eloe

eloe koesoe

hoesoe

aloehoe.

ahanene

assa

toe

)

akene asinilo

toekanneli

katawa

pasieloepea

likatawa

ija

posso

posso

ipoenanoea

ihahoe

sele

orasa

oehoewa

inkana

)

》)

oelowalare

panene

tahaija

mememie

atak

kalaka

loelina

kekeloe

ewoe sahaeë

oeloh

reneh oelot

oehei

loewa rene

anene

hasa

arana hale

amole hilale

kapile
U L I A S S E R S.

ASSiloeloE. NOESA LAUT. SAPAROEA. HAROEKO:

\begin{tabular}{l|l|l} 
ipasanelie & poso (epakylo) pakelo & oepake
\end{tabular}

toekane ma- toekan obat hetewa oso ake obate

kaoena aji- oebat

wakal

pietahoe-

maisy

strasele nase- lenemay

nya

pakewoewaka

pietahoemai- tenoe

sy

poseke

papata

anaanape-

hene

apoena oe-

oewa

hawoe

sele

oenarahoe

kwatty ata-

roe

kekahoesele

haoeltitirinnjo

)

manana

soloko

makanna

hoewako

oesoel

oehoewaane-

hia

hoea

hoëoewo

ipaneneta

haijani

poene sala

oeloekoekete. hoetoewako

ne oegejtee

otake

palakaa

atoeloenge

ketasalano

kewoe raha-

hie

oeloe

oeloekoe

ketane

kaoese

loemaka

panene

opakej oebat

allahae heti-

tolo

heil

)

nanai

hamoeële

wako wako

soepoe loeka

moetery

allamoemoe

epoetoe

ehety hatoeil

soloko

makana

oesoko

oesoey eloeroëlo

hoeoewo

onnoe ema-

kana

pisarata se-

hoka

roenie oello oeroehoëtino

senawakko

tahary

sroeamaoeil

aymasele

oeroeny

roenymasele

ahoese

roekoerako

poenneë

hasa lamoelili ahoeë

arana helale

pouloheenoe koeing

moley "aka-

loene

arene tahary

toeroesaa

oroew

oroew ehety

hoese

noekoeraoew

yonnoej

seej oebat

poloej"

ekey oepahalae

n)

banka

poenabyasa

saekoene

palamomoe

paheka pa-

heka

strasela hatoe

strakana

oesoey

oesoehela

keoerre

olaay

mokoeyeala

oetak

maatoelang

koerapo

ole ole

oeroey

oeromaselet

ahoesel

makari

hoeta klening

imakoeaoew

ninoe

kolo aymoeato

roroew

kakey 


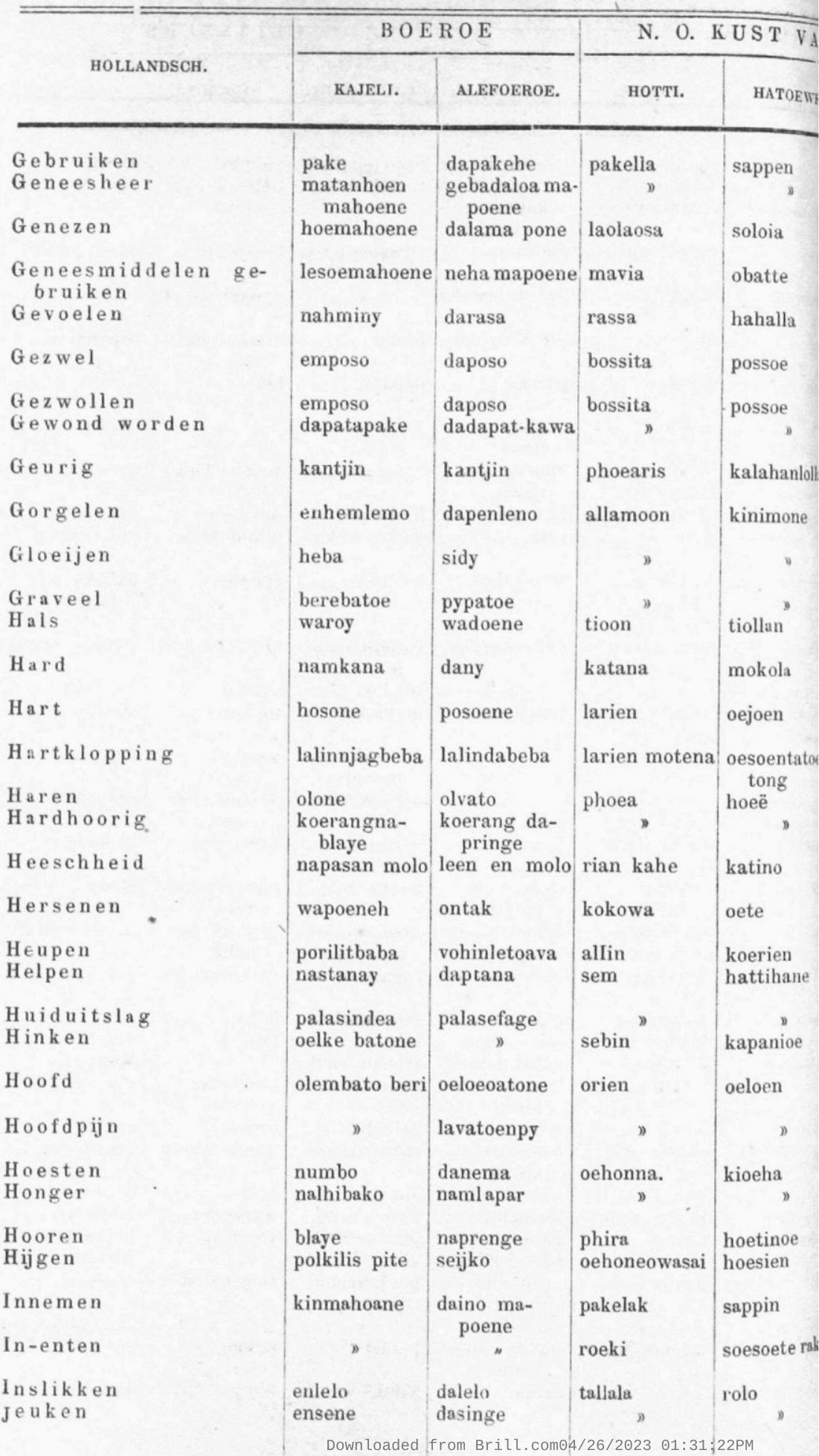




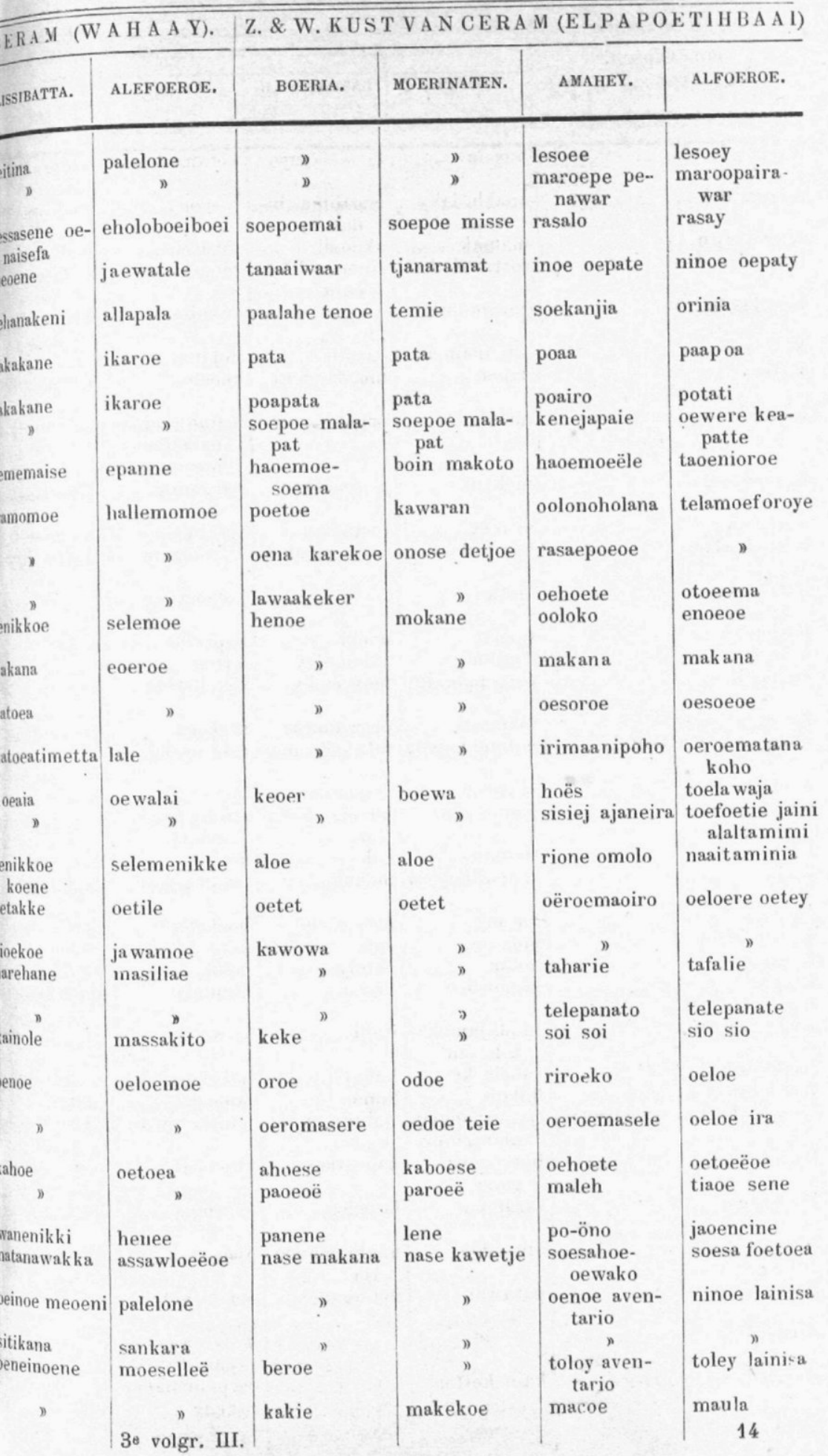




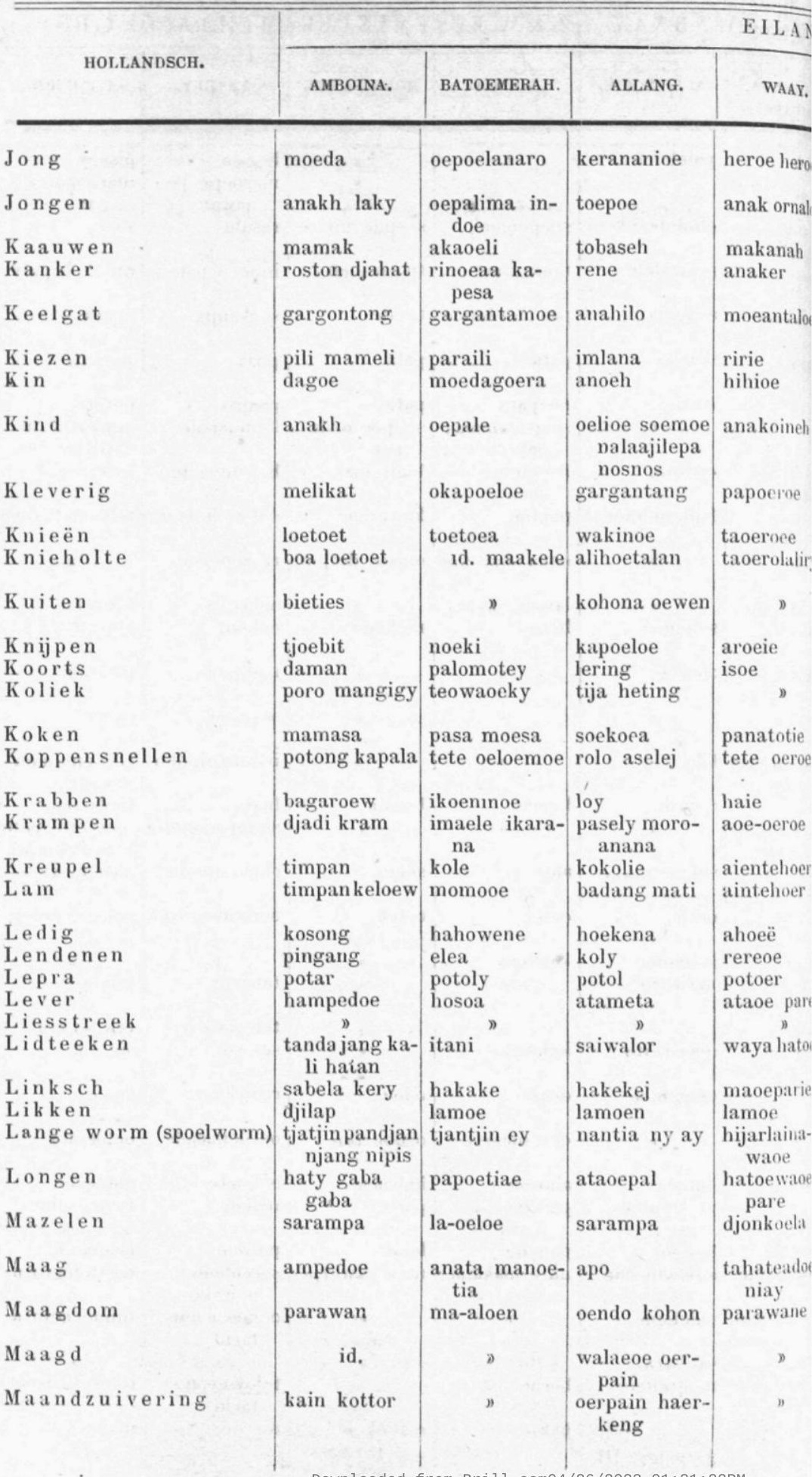




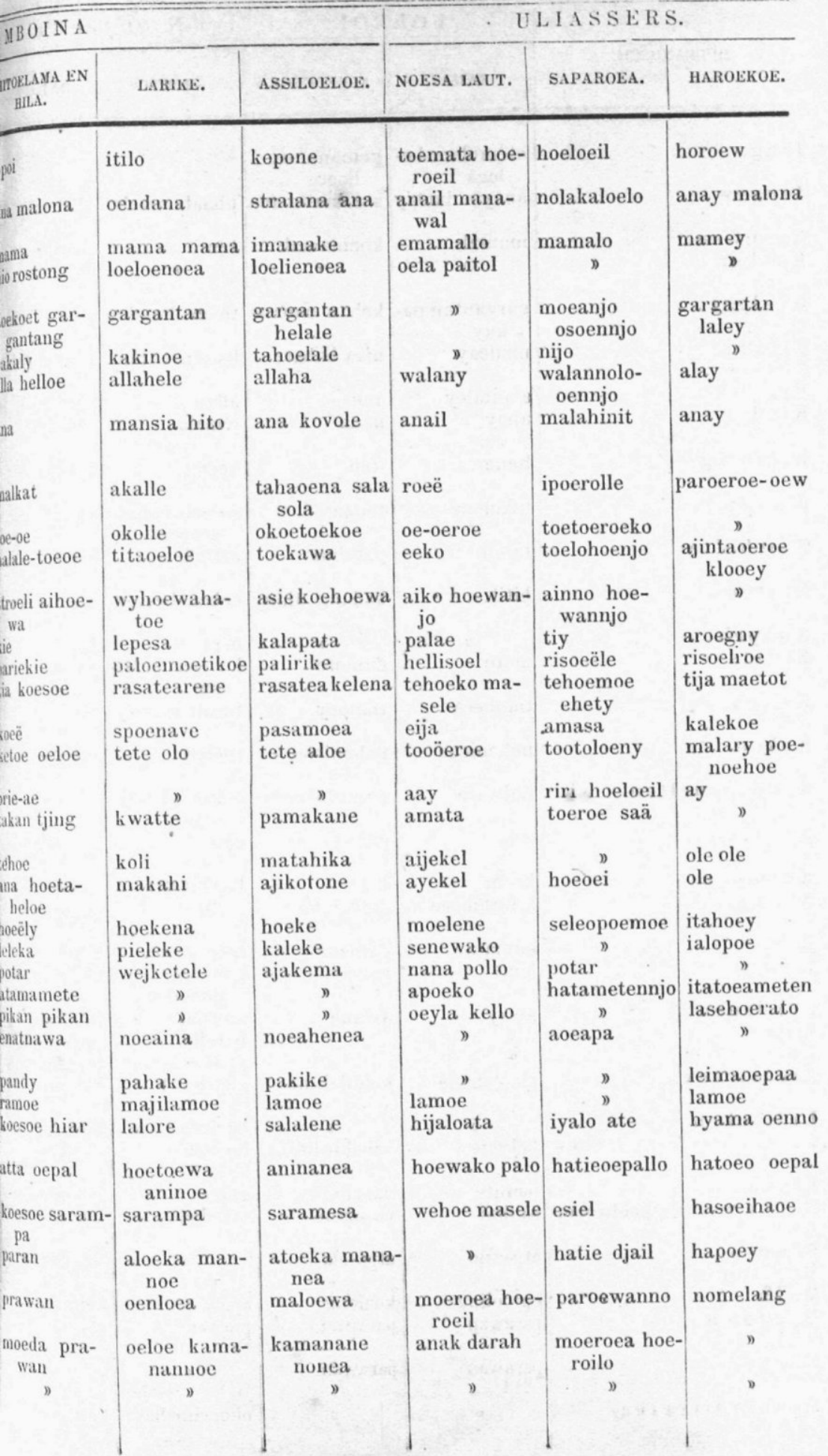




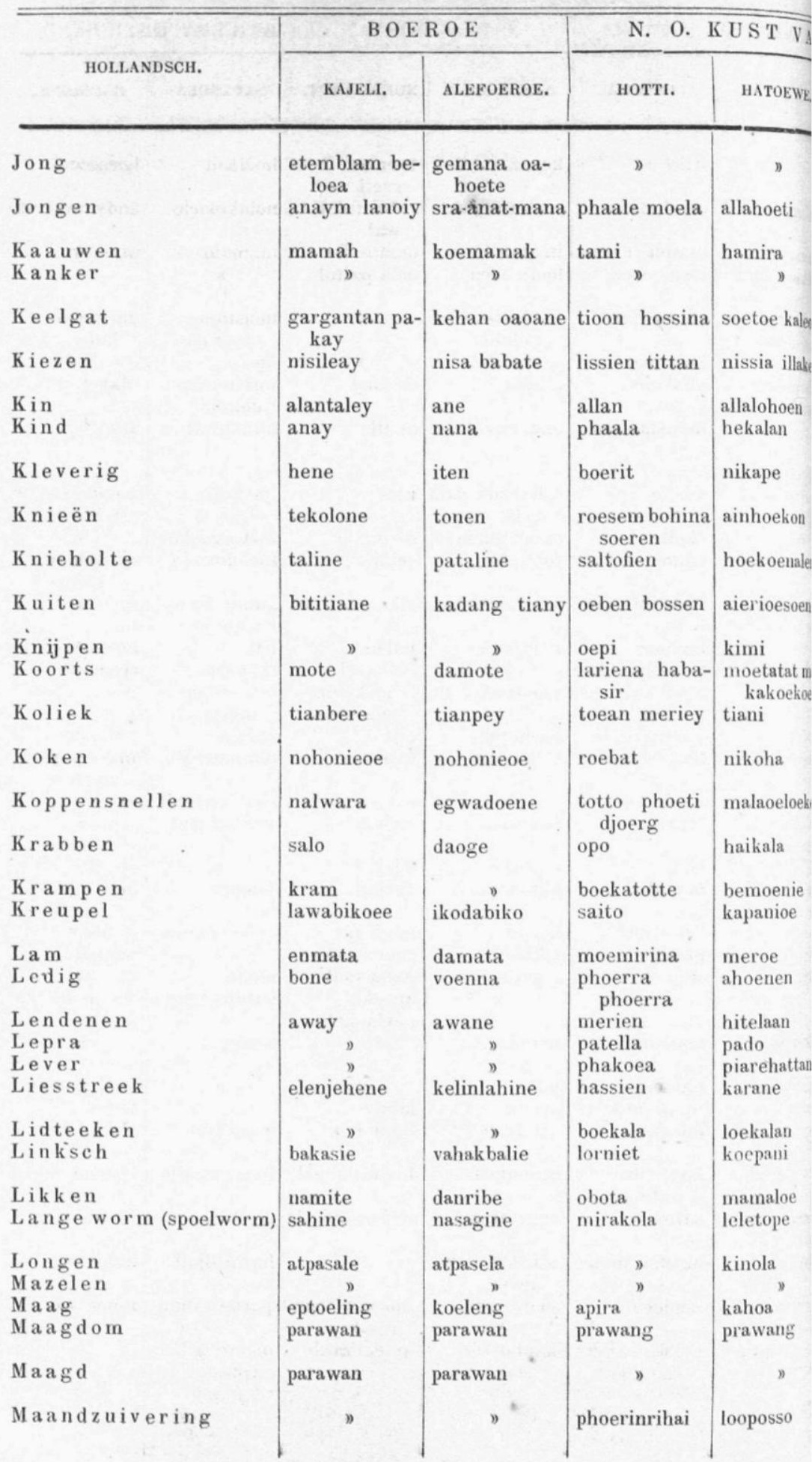









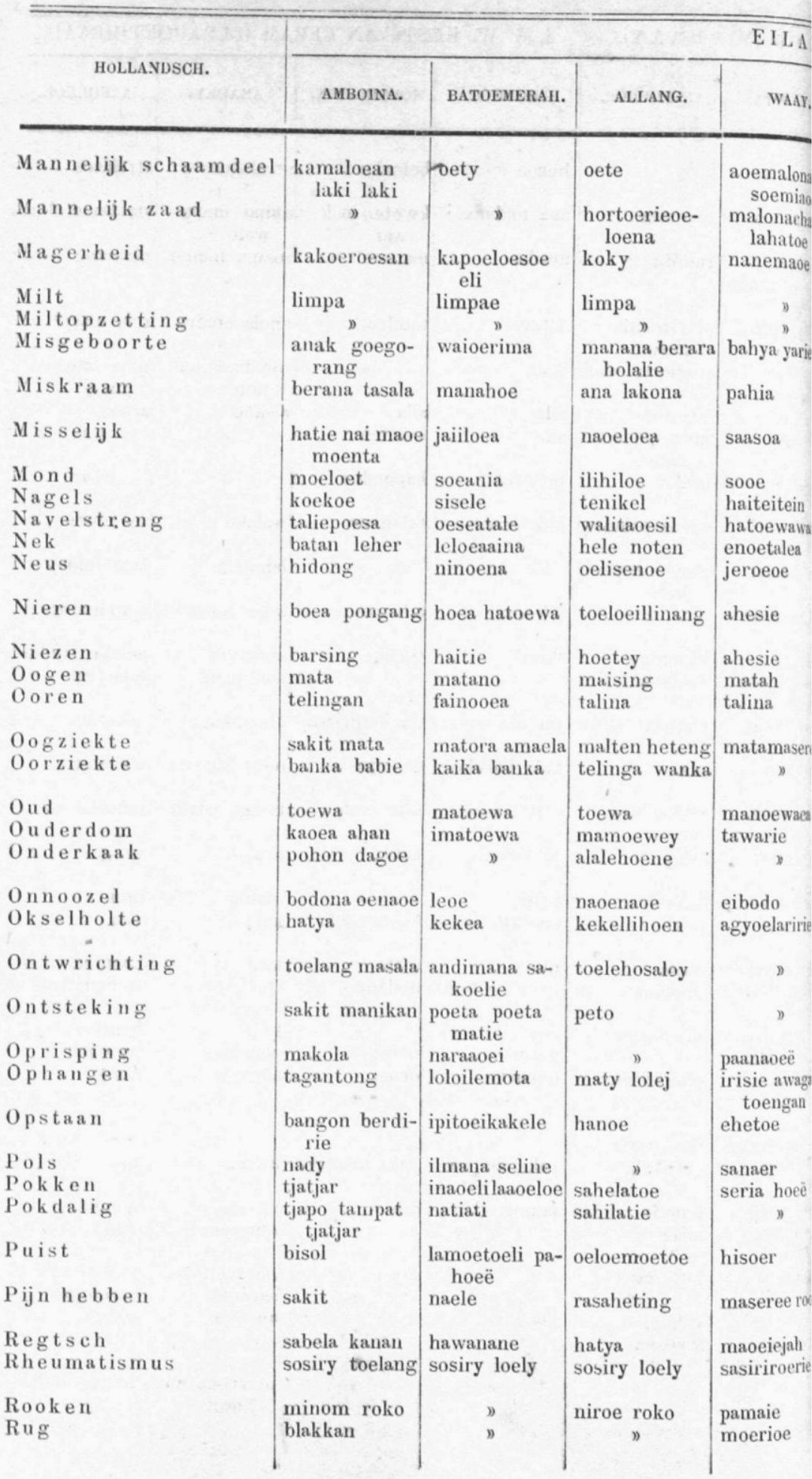




\section{BOINA.}

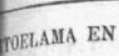
HILA.

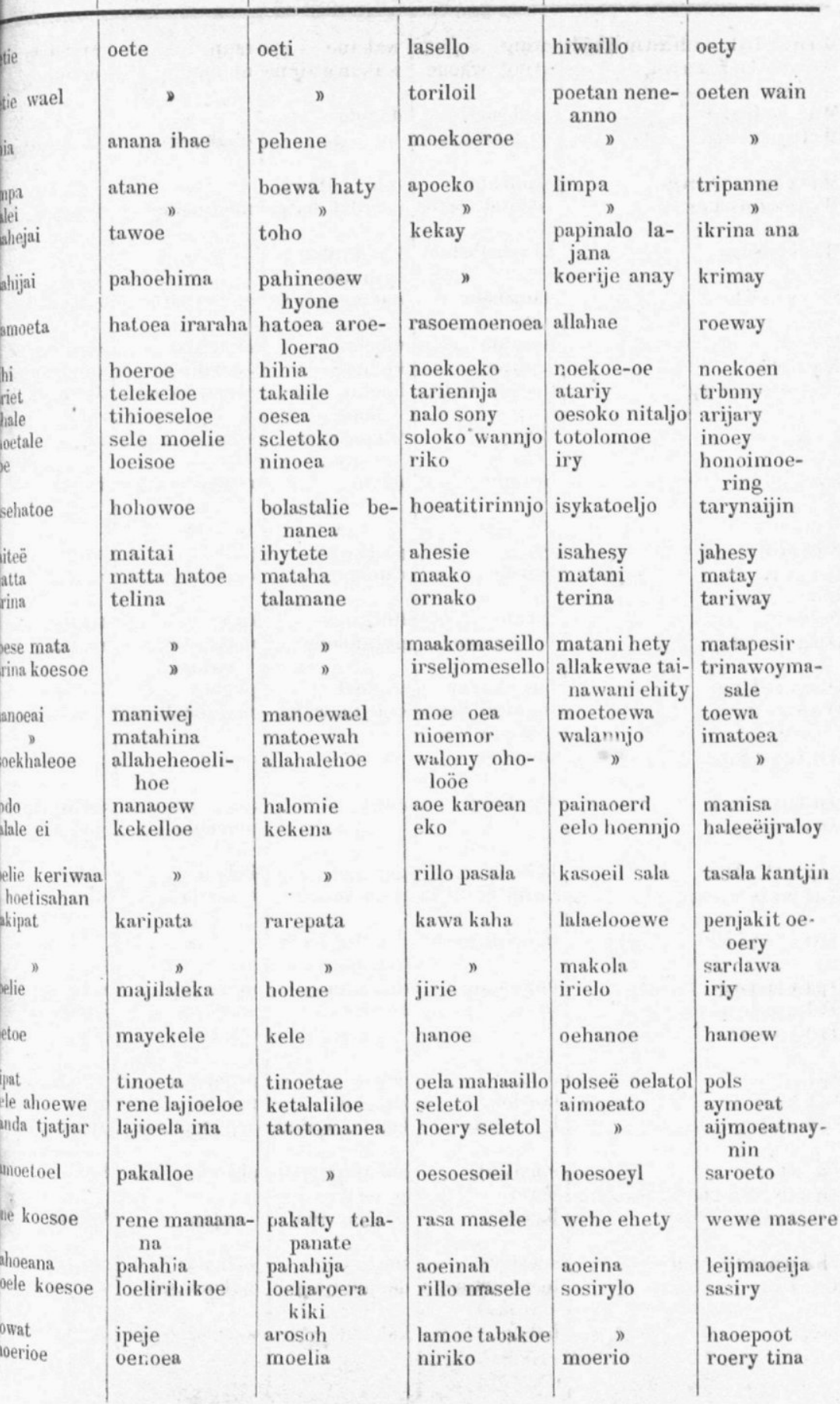




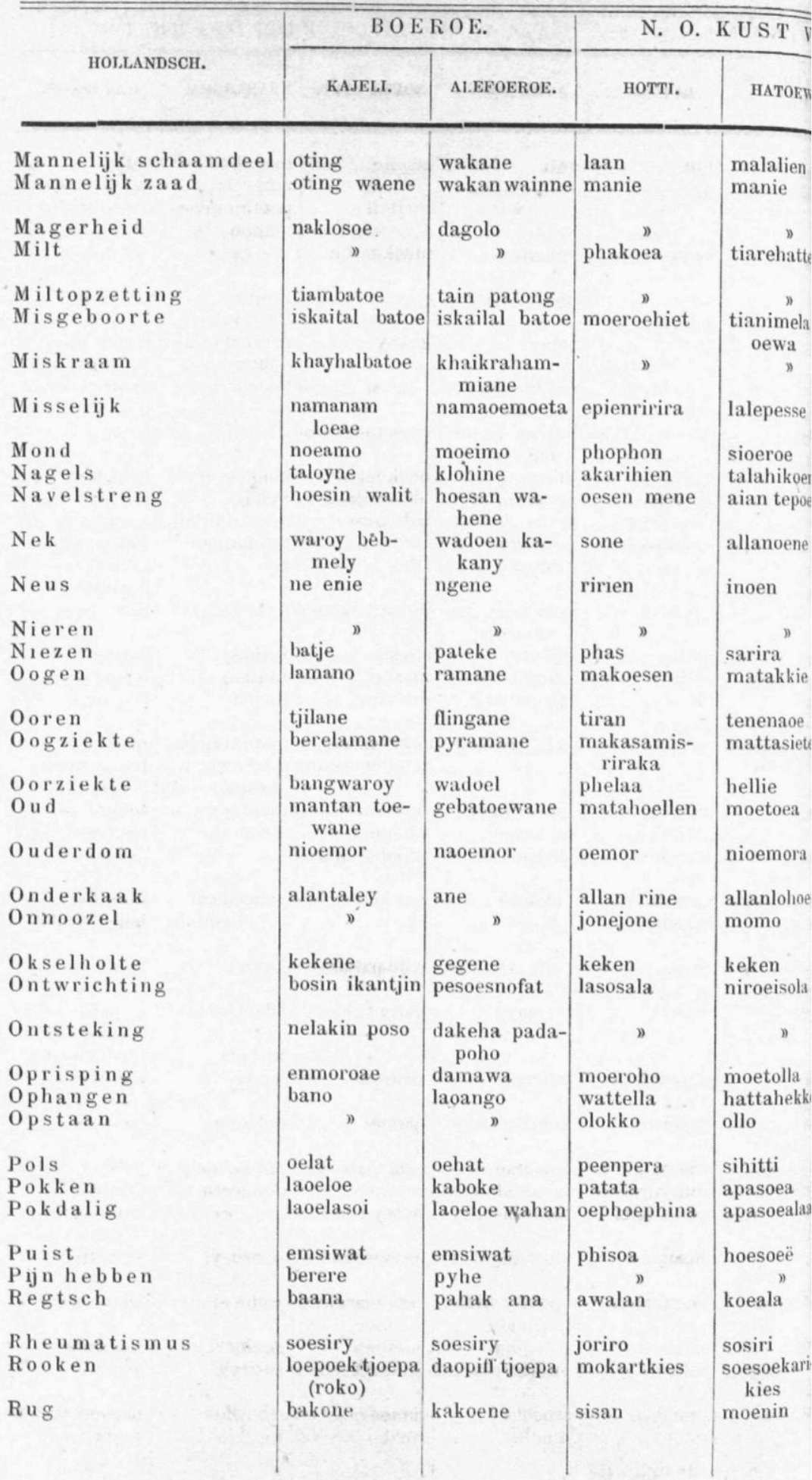




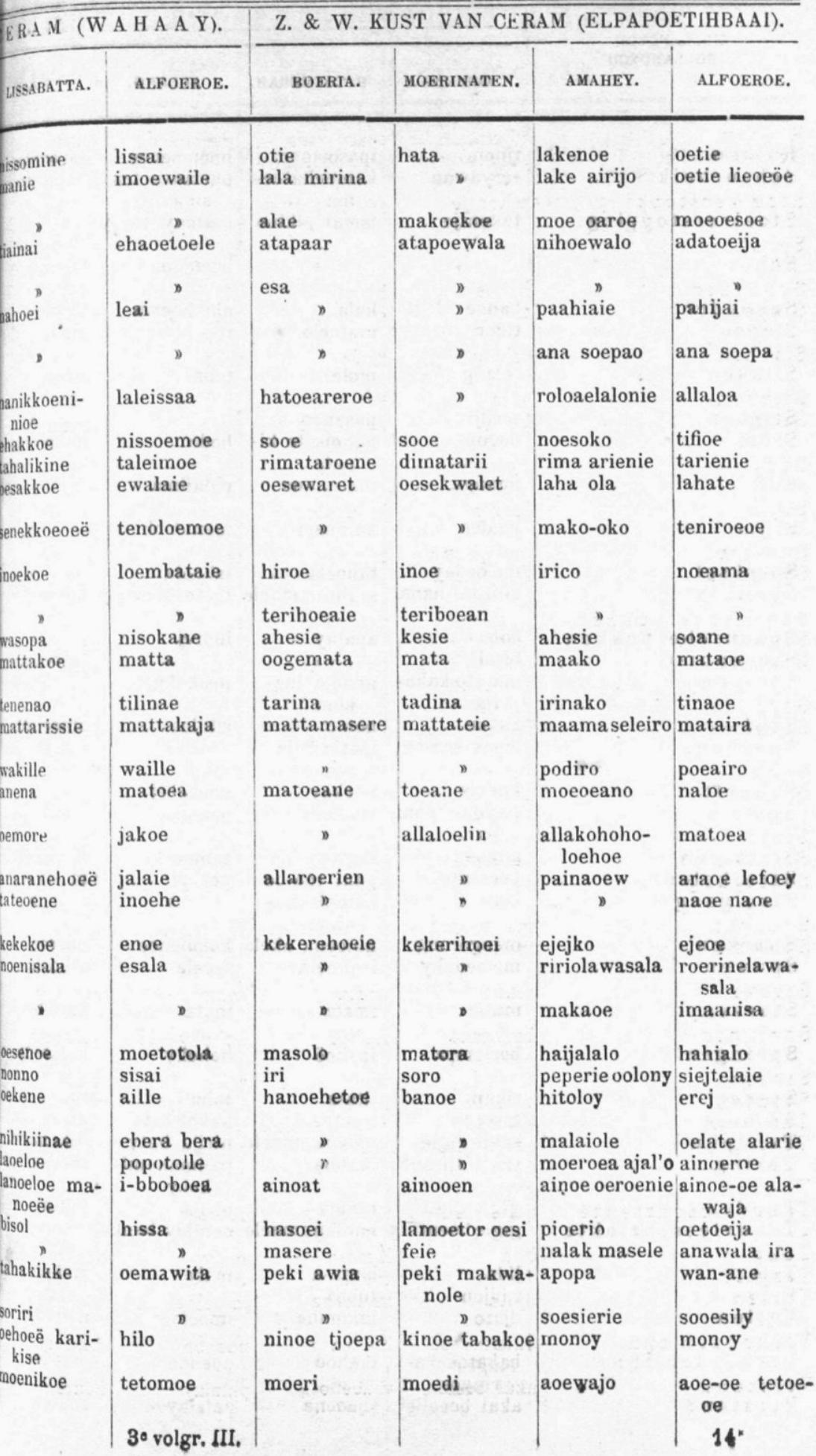




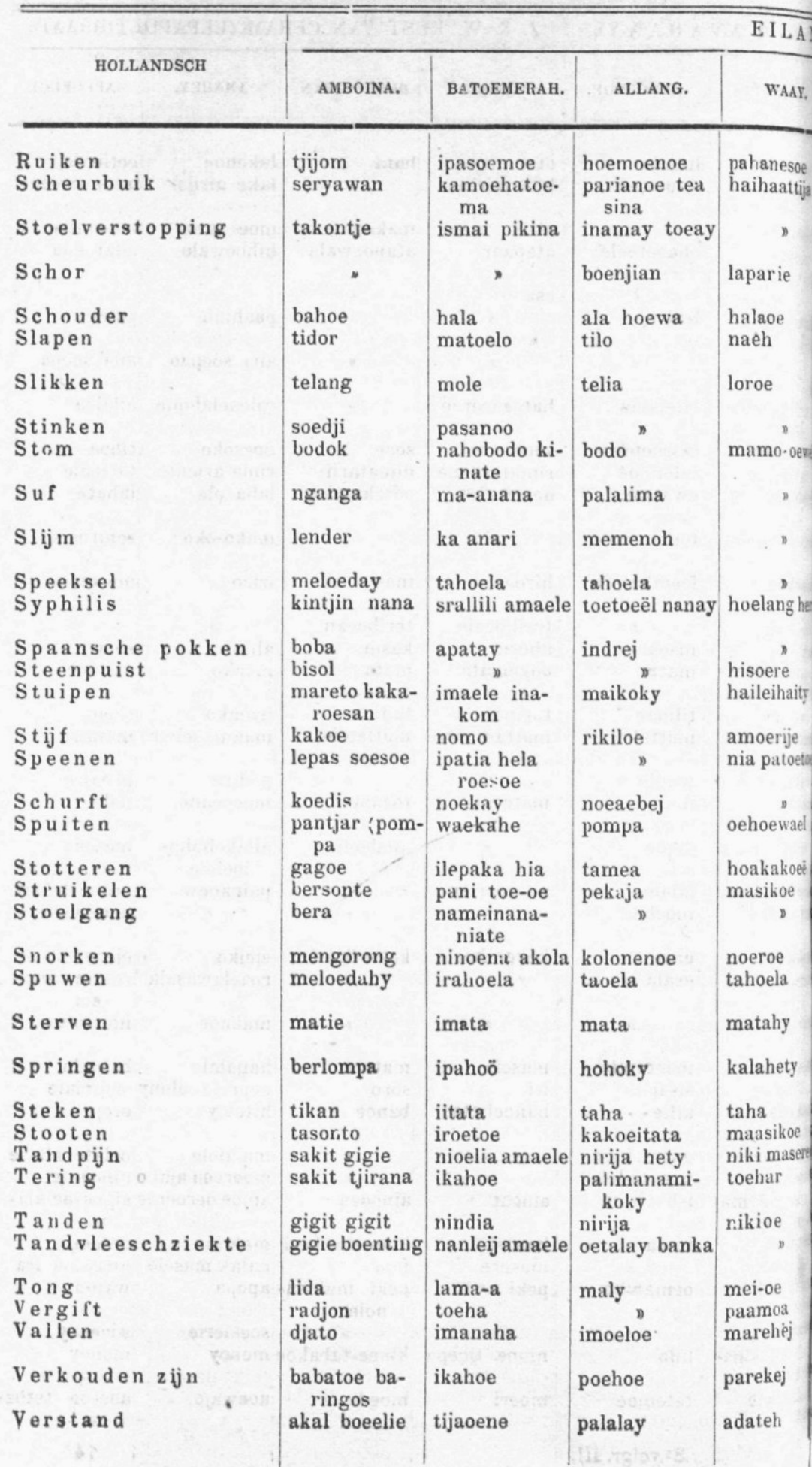




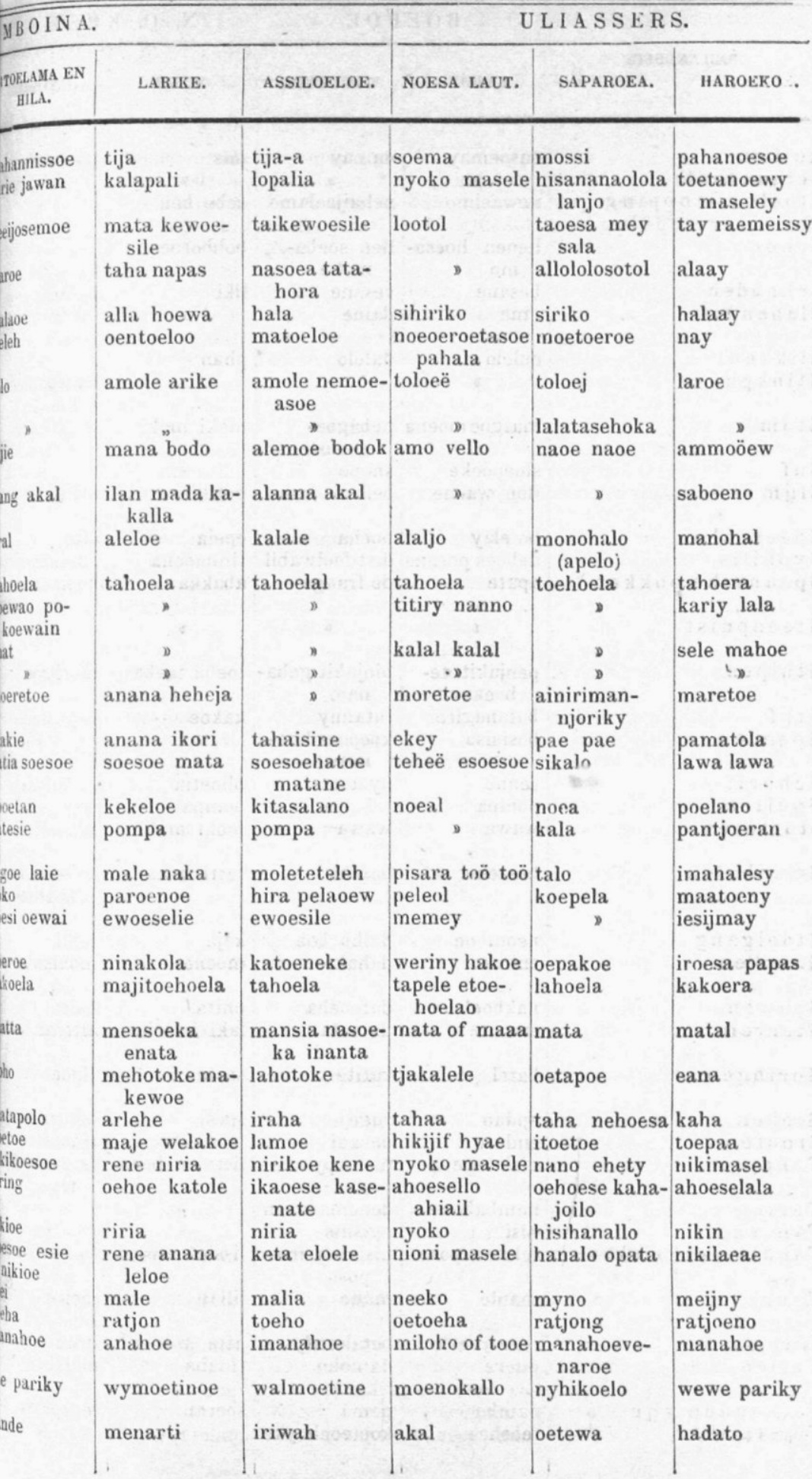




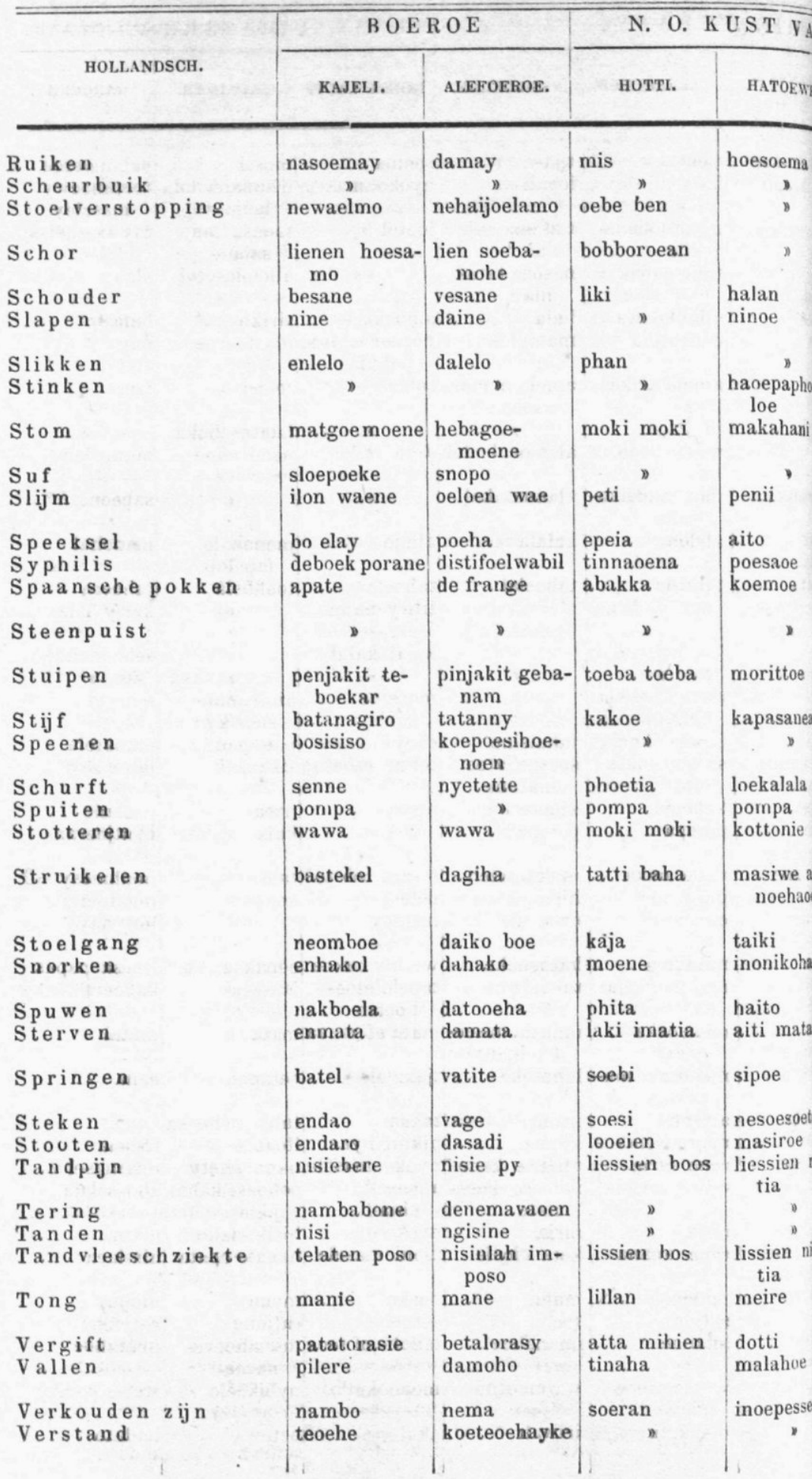




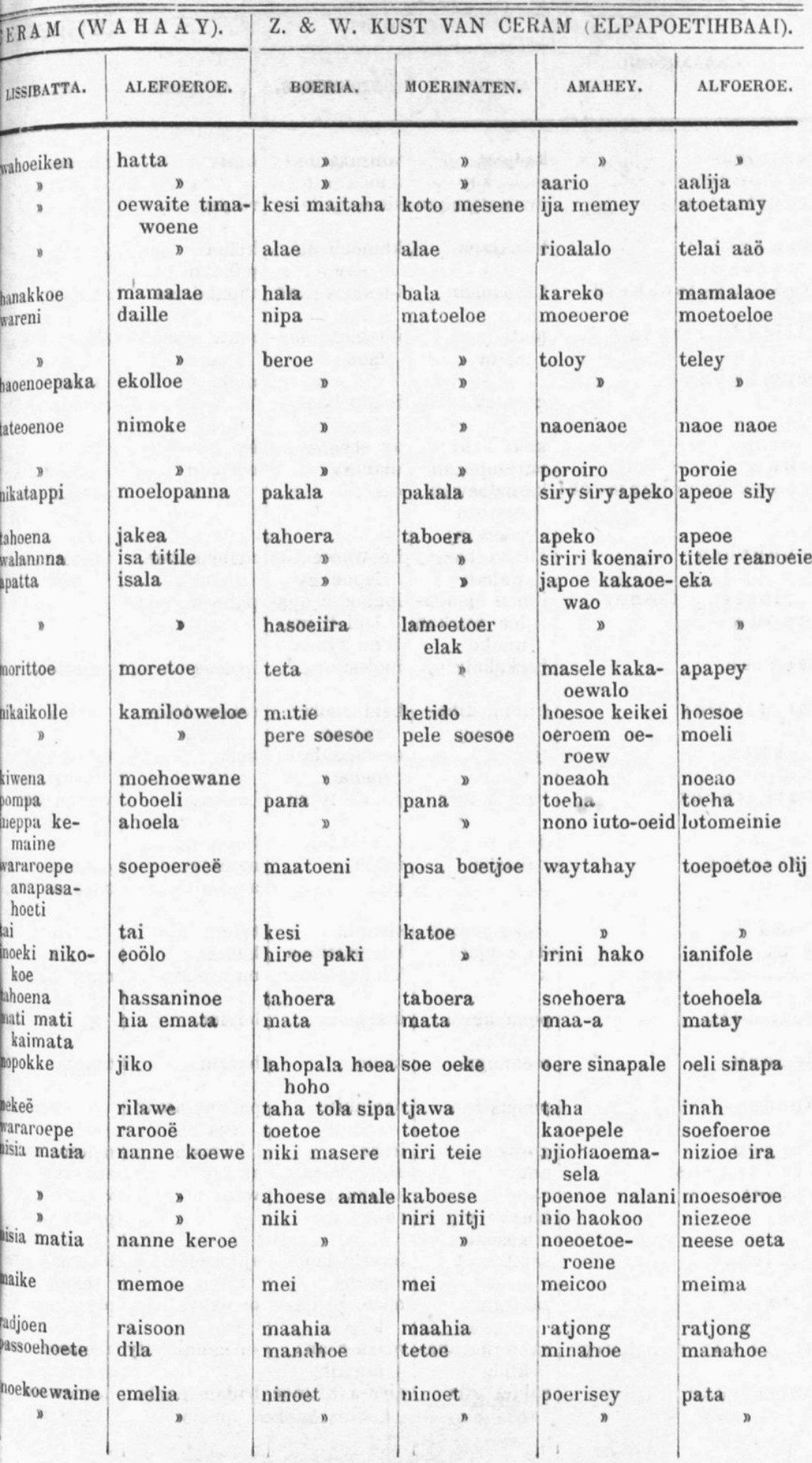




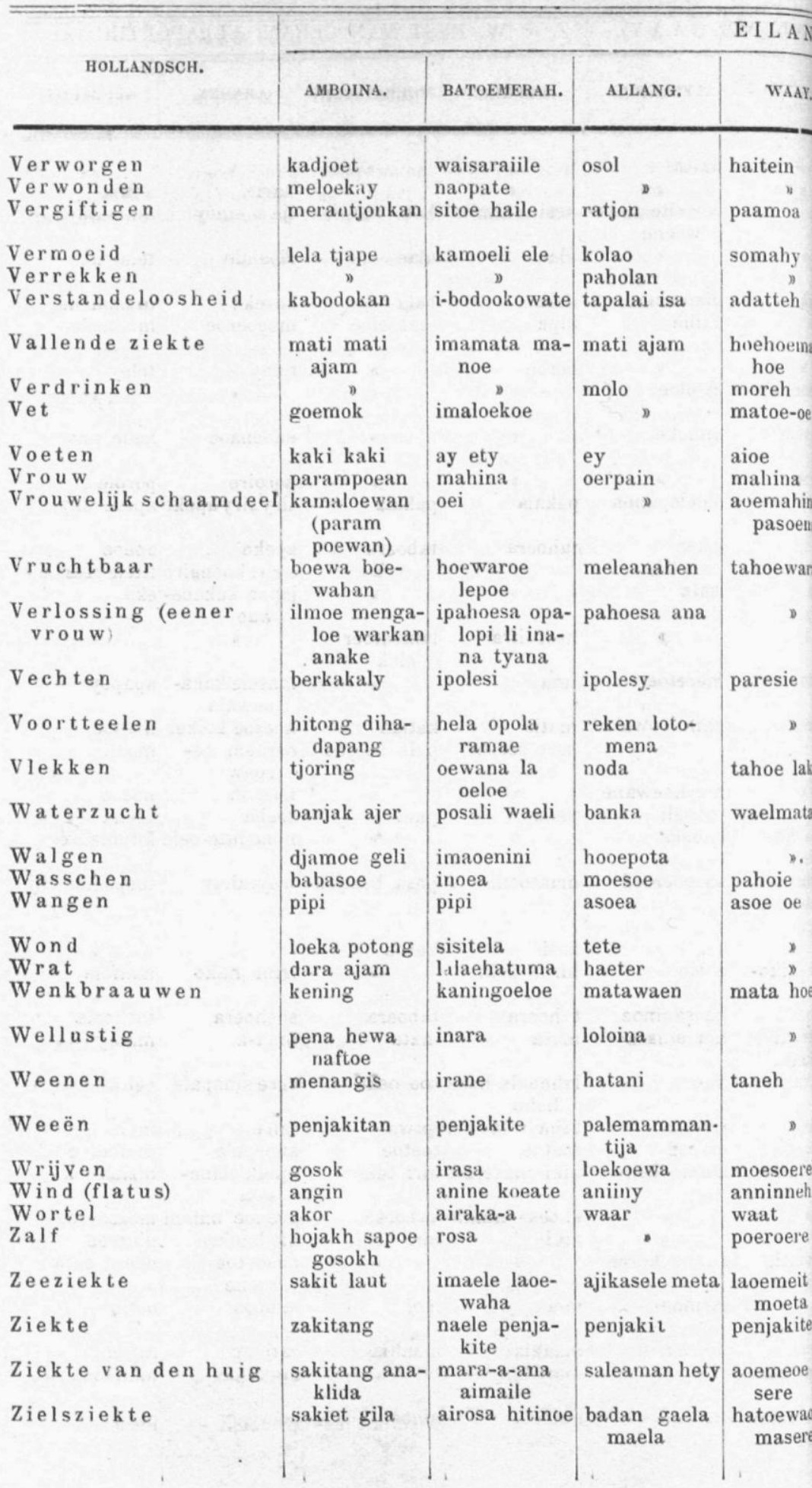




\section{A I BO I NA.}

MTOELAMA EN HILA.

LARIKE.

ASSILOELOE. NOESA LAUT.

mekari

patanoie

asoe oe

enat

hala

hatoea taoet naftoe

soesa

tekene

kosot

anin

aivaat

2 in

besoe meid

$$
\begin{gathered}
\text { roes } \\
k i \\
\text { knes }
\end{gathered}
$$

soesoe palalae

voesoe ma-

maoe asarama

aroehama

pehe

mase patale

manoea tahi-

boedy

mema

ninon weloe

oenloekoe

wejoeroe

roepae

oewe noty

mai bowatte

oercepae makalajana

main hoen-

loema

miranjanej

laoe

posoposo welloe

lapoma

meroeoely

ikika

isoele

toewahnie

pehi

oemoerijko

apata lijsele lamoe

manoesia ta-naoenaoennjo poroilo

haboedy

mamemate

ninoe wael

mawekoe.

ajimanssa

mahina

oei

masele ma-

noea

molo ma-a

moesene

aijiko

pipinal

poeki jollo

baroe op hoe- oehawa

wa

makalaja ma-

hina

aoew

tika loeno

wajille ara-

hani

anisoe

lopo

haika-a

noesa

mana iginne

majikolo

mekolo

majirotaki

anini

waana

oeloeta

bere makana- kitanilaoe

lihaha

moeraty

haha

moeratty

ajam

noroel

oeha

n

laijana

koerinallo

nariginne

ilane

irane

irosake

anine

wakarroew

apoeloe

haha haha arima
U L I A S S E R S.

SAPAROEA. HAROEKOE. noewah

kaihoeoew n

poetannjo ikehaa

ratjong eloperatjoen

koaka

tasale oerato

naoew naoew

mamemal

matymati

oemata moko matakasoy

moeisy minaa

aiko ajinwey

papinalo mahina

ijole oenooe- oey

haeanapelae

ahoeanne

poenabianno

poea of toewa

poetaneë

panoe panoe

poewa waello waello waeori- pika pika ay

\begin{tabular}{l|ll} 
moesay & $\begin{array}{l}\text { toloke } \\
\text { kowa }\end{array}$ & pakoe poesi
\end{tabular}

poea of toewa amaeise patanoy

\begin{tabular}{l|l} 
wasoeko passoe poe ë & patoe poen
\end{tabular}

poetay $\quad$ malapa

hoeteljo dara ajam hoetiro

noesa ae pariano mata oeweri- iwanno

kiky soeka niy telo

kalea-a japa kany

masele lijano allahae masele

\begin{tabular}{l|l|l} 
soes ay & sosae
\end{tabular}

kersa-o aninno anino

waeljo waallo awar

moesery laoe- moeserylaoe- masele laoeti

maseleyllo manoehalo penjakite

\ anahoeileije meini amy ma-

roemtaie ma- pamoeko maoekele 


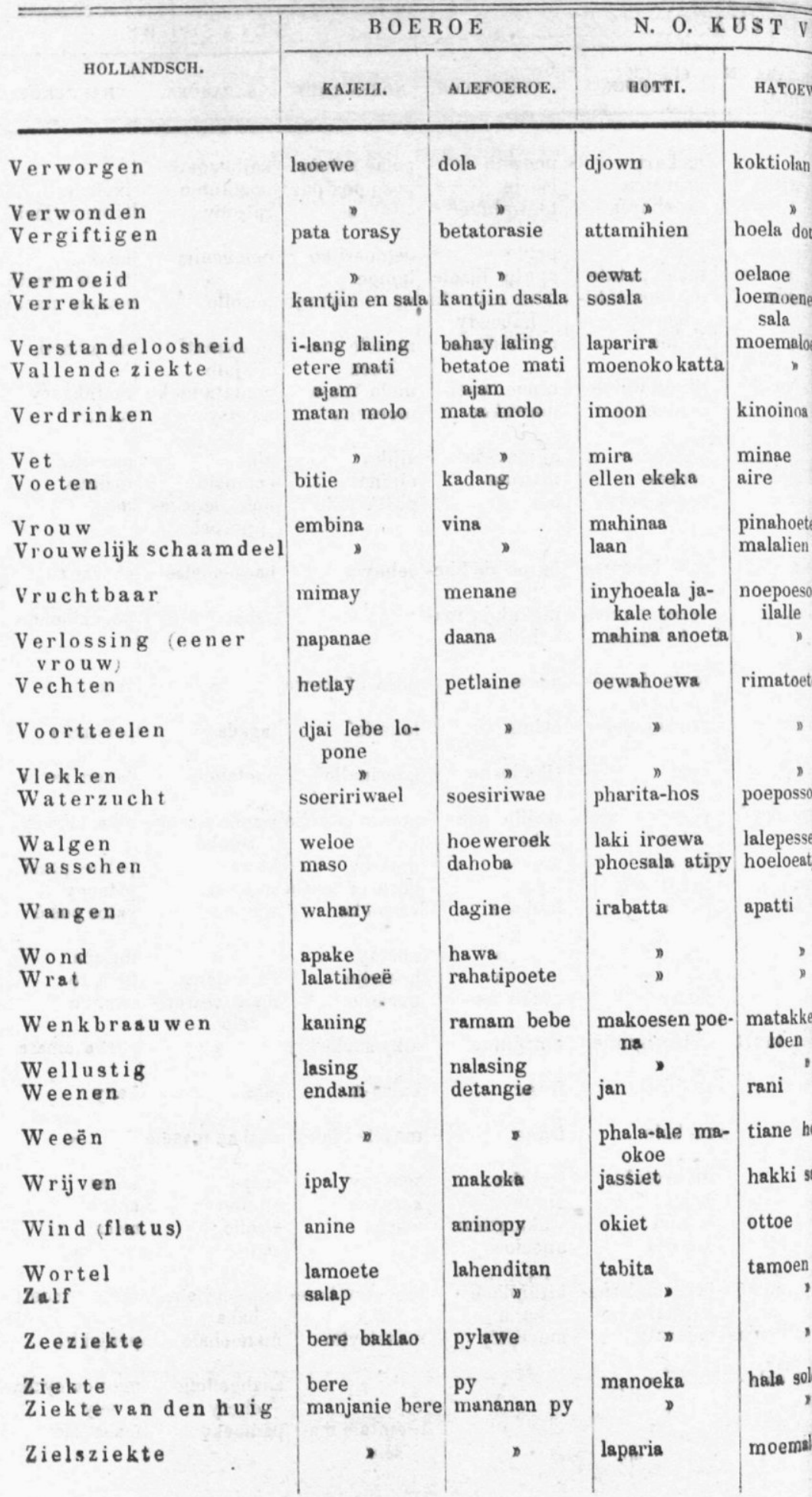




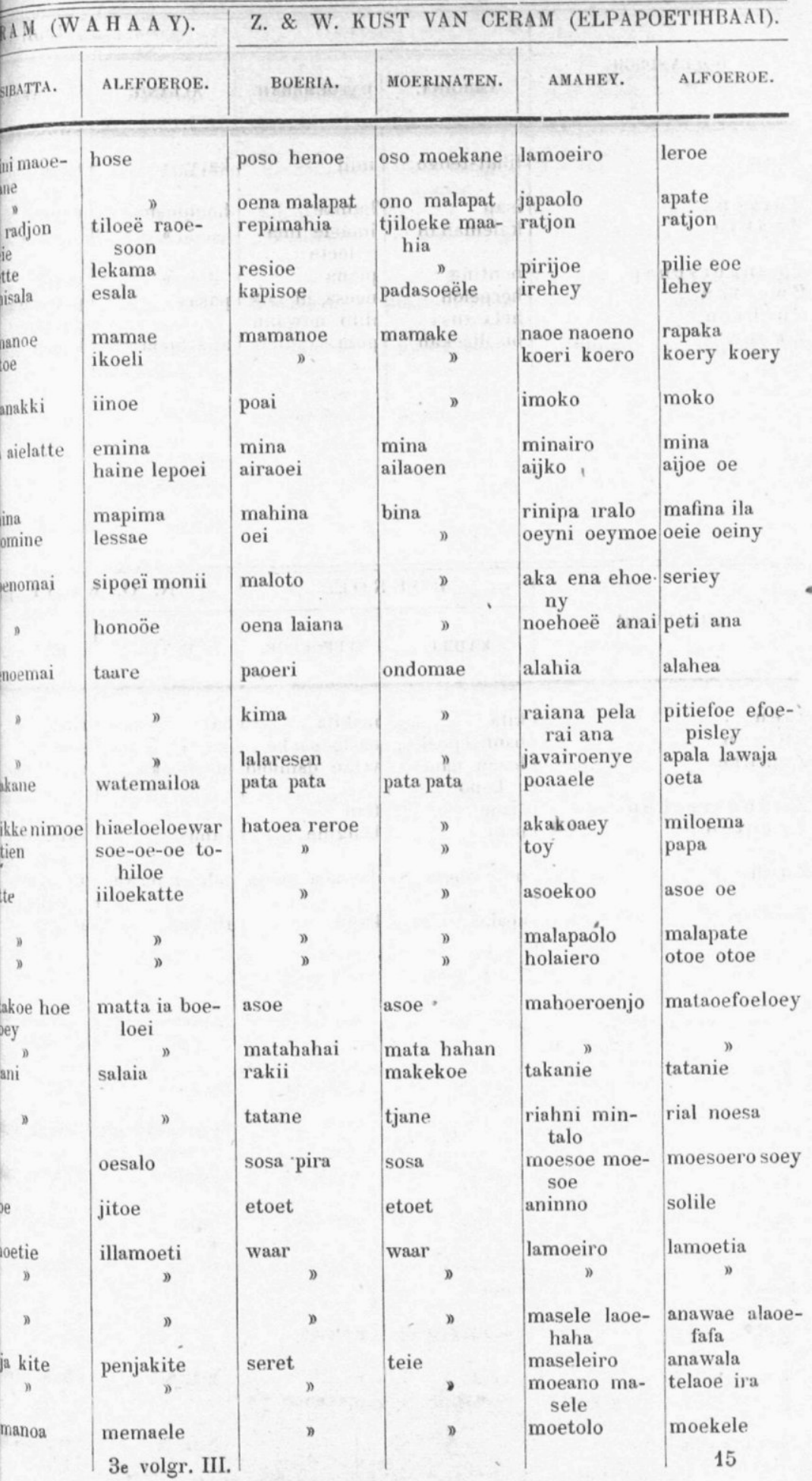




\begin{tabular}{|c|c|c|c|c|}
\hline \multirow[b]{2}{*}{ HOLLANDSCH. } & \multicolumn{4}{|r|}{$\overline{E I L}$} \\
\hline & A MBOINA. & BATOEMERAH. & ALLANG. & \\
\hline Zien & lihat-tengo & imii & kary & naoe \\
\hline $\begin{array}{l}\text { Zuigen } \\
\text { Zwakte }\end{array}$ & $\begin{array}{l}\text { isap } \\
\text { kalemahan }\end{array}$ & $\begin{array}{l}\text { hamoe } \\
\text { imaele ima- }\end{array}$ & hoenioenoe & pamay \\
\hline $\begin{array}{l}\text { Zwangerschap } \\
\text { Zweeten } \\
\text { Zuchten } \\
\text { Zweer }\end{array}$ & $\begin{array}{l}\text { bonting } \\
\text { berpeloh } \\
\text { hela susa } \\
\text { bisolloekah }\end{array}$ & $\begin{array}{l}\text { loeta } \\
\text { piana } \\
\text { noesa ili } \\
\text { ilihi nawane } \\
\text { pota }\end{array}$ & pasay & $\begin{array}{l}\text { tyaie } \\
\text { panatie } \\
\text { palahlie } \\
\text { hisoer }\end{array}$ \\
\hline
\end{tabular}

\begin{tabular}{|c|c|c|c|c|}
\hline \multirow{2}{*}{ HOLLANDSCH. } & \multicolumn{2}{|c|}{ B O E K OE. } & \multicolumn{2}{|c|}{ N. O. KUST } \\
\hline & KAJELLI. & ALEFOEROE. & нотті. & НАTOE \\
\hline $\begin{array}{l}\text { Zien } \\
\text { Zuigen } \\
\text { Zwakte } \\
\text { Zwangerschap } \\
\text { Zweeten } \\
\text { Zuchten } \\
\text { Zweer }\end{array}$ & $\begin{array}{l}\text { kita } \\
\text { nanhoepoeke } \\
\text { batan nam- } \\
\text { bono } \\
\text { tiane } \\
\text { pani } \\
\text { nese soesa } \\
\text { apake }\end{array}$ & $\begin{array}{l}\text { makita } \\
\text { dasloepoeke } \\
\text { vatan damlolo } \\
\text { tian } \\
\text { bangihe } \\
\text { daesana soesa } \\
\text { kawa }\end{array}$ & $\begin{array}{l}\text { ha } \\
\text { isoesi } \\
\text { moenoeka } \\
\text { lahassa } \\
\text { kahas } \\
\text { oehoen nawa } \\
\text { abakka }\end{array}$ & $\begin{array}{l}\text { rinei mo } \\
\text { soesoa } \\
\text { moeloen } \\
\text { ollo hoel } \\
\text { kalessen } \\
\text { poela } \\
\text { aselamoe } \\
\text { kakka } \\
\text { apattı }\end{array}$ \\
\hline
\end{tabular}


